\title{
PRODUCTION OF UNPURIFIED AGAR-BASED EXTRACTS FROM Gelidium sesquipedale SEAWEED BY MEANS OF SIMPLIFIED EXTRACTION PROTOCOLS
}

Marta Martínez-Sanz ${ }^{1 *}$, Laura G. Gómez-Mascaraque ${ }^{2}$, Ana Rosa Ballester ${ }^{3}$, Antonio Martínez$\mathrm{Abad}^{4}$, Andre Brodkorb ${ }^{2}$ and Amparo López-Rubio ${ }^{1}$

${ }^{1}$ Food Safety and Preservation Department, IATA-CSIC, Avda. Agustin Escardino 7, 46980 Paterna, Valencia (Spain).

${ }^{2}$ Teagasc Food Research Centre, Moorepark, Fermoy, Co. Cork, Ireland

${ }^{3}$ Food Biotechnology Department, IATA-CSIC, Avda. Agustin Escardino 7, 46980 Paterna, Valencia (Spain).

${ }^{4}$ Dept. Analytical Chemistry, Nutrition and Food Sciences, University of Alicante, 03690, San Vicente del Raspeig, Alicante, Spain

*Corresponding author: Tel.: +34 963200022; fax: +34 963636301

E-mail address: mmartinez@iata.csic.es 


\begin{abstract}
This work reports on the production and characterization of agar-based extracts from the seaweed Gelidium sesquipedale by means of simple protocols based on hot-water and sonication treatments. The combination of sonication with the hot-water treatment reduced 4-fold the extraction time without significantly affecting the extraction yield (ca. 10-12\%) and the extracts' properties. Apart from agar, the extracts contained proteins, polyphenols and minerals, which conferred them high antioxidant capacity and led to the production of brownish softer gels. The application of an alkali pre-treatment yielded almost pure agars, with higher molecular weights and crystallinities than commercial agar, resulting in stiffer gels. However, the partial digestion of agar by the alkali led to low extraction yields (ca. 2$3 \%)$.
\end{abstract}

These results show the efficiency of the combined heat and sonication method to generate cost-effective agar-based extracts with potential applications within the food industry.

Keywords: seaweed; gels; sulphated polysaccharides; X-ray diffraction; food hydrocolloid 


\section{Introduction}

Agar is a polysaccharide that constitutes the main structural component in the cell walls of certain species of seaweed belonging to the Rhodophyceae (red algae) class. Amongst the different seaweed species available for agar extraction, Gelidium sesquipedale is the most widely used raw material in Spain and Morocco (Carmona, Vergara, Lahaye, \& Niell, 1998; Mouradi-Givernaud, Hassani, Givernaud, Lemoine, \& Benharbet, 1999) and it typically produces agars with greater gelling capacity than those obtained from Gracilaria species (Pereira-Pacheco, Robledo, Rodríguez-Carvajal, \& Freile-Pelegrín, 2007). Agar is constituted by two major components: agarose and agaropectin. Agarose is the gelling fraction of agar and consists of repeating units (agarobiose) of alternating $\beta$-Dgalactopyranosyl and 3,6-anhydro- $\alpha$-L-galactopyranosyl groups. Agaropectin has a similar structure but contains 5-10\% sulphate esters and other residues, such as methoxyl groups and pyruvic acid, in addition to polysaccharide chains in which the 3,6-anhydro- $\alpha$-Lgalactose units are partially or completely substituted by $\alpha$-L-galactose 6 -sulphate units (Sousa et al., 2012; Venugopal, 2016).

The type, amount and location of these substituents strongly affect the physical properties of agar (Freile-Pelegrın \& Murano, 2005). Upon solubilization in hot water, and under specific conditions, agar forms a slightly viscous fluid that can form a thermoreversible gel when the temperature is decreased below a certain point (i.e. gelling temperature). The agarose component is critical for the gelation of agar. The gelation mechanism of agar has been hypothesized to take place through a two-step mechanism. The first step consists of a transition from a fluctuating, disordered coil conformation in solution to a rigid, ordered structure which forms the junction zones of the gel network (Mohammed, Hember, Richardson, \& Morris, 1998). Additionally, under certain conditions, these helices can aggregate to form thick bundles, leading to the formation of strong gels. Many factors, such 
as the agar concentration, the amount and type of impurities and the cooling rate, may affect this gelation mechanism (Aymard et al., 2001; Lai \& Lii, 1997; Lee, W.-K. et al., 2017; Nishinari \& Watase, 1983; Watase \& Nishinari, 1983).

Agar is widely used in the food industry area (mainly as texture modifying and thickening agent) and in the microbiology field. However, alternative applications such as the development of biodegradable films (Atef, Rezaei, \& Behrooz, 2014; Guerrero, Etxabide, Leceta, Peñalba, \& De la Caba, 2014; Kanmani \& Rhim, 2014; Phan, Debeaufort, Luu, \& Voilley, 2005; Rhim, 2011), hybrid biopolymeric nanofibers (Sousa et al., 2015), encapsulation structures (Alehosseini et al., 2018) or components with bioactive functionalities (such as antioxidant and antitumoral properties) (Chen, H.-M., Zheng, \& Yan, 2005), are currently being explored. Depending on its aimed application, agar must possess a different range of properties (i.e. different gel strengths, degree of purity, colour, etc., may be required). For instance, while high quality agars with gel strengths greater than $700 \mathrm{~g} / \mathrm{cm}^{2}$ (in 1.5 wt.-\% solution) are often required by the international market for microbiology applications (Armisen, R., 1995), agars with lower gel strength values (30$200 \mathrm{~g} / \mathrm{cm}^{2}$ ) may be preferred for applications within the food industry (Pereira-Pacheco et al., 2007). The properties of the agar are strongly determined by the raw seaweed species used and by the protocol applied for the extraction process, since these will affect the chemical composition and molecular weight of the extracted agar (Pereira-Pacheco et al., 2007). The industrial process for agar extraction involves the application of alkaline pretreatments, followed by time- and energy-consuming high-temperature and high-pressure treatments, filtration processes at high temperatures and several freeze-thawing cycles to eliminate the water (Armisen, Rafael \& Galatas, 1987). The influence of extraction temperature and extraction time on agar characteristics has been reported for Gracilaria species. A notable reduction on agar yield and decrease in gel strength were observed for 
elevated extraction temperatures and prolonged extraction times (Arvizu-Higuera, Rodríguez-Montesinos, Murillo-Álvarez, Muñoz-Ochoa, \& Hernández-Carmona, 2008). Furthermore, the application of alkaline pre-treatments has been seen to be critical for obtaining good quality agars from Gracilaria species. However, the pre-treatment typically leads to a significant decrease in the extraction yield and the conditions have to be optimized to prevent a strong degradation of the agar (Meena, Prasad, Ganesan, \& Siddhanta, 2007). As an alternative to the conventional method, exploring simpler extraction processes based on combined heat and ultrasound treatments would enable reducing the amount of time and energy needed. Ultrasound-assisted extraction has been reported to be an efficient strategy for the extraction of diverse compounds such as lipids (Prabakaran \& Ravindran, 2011), proteins (Bleakley \& Hayes, 2017), polyphenols (Adjé et al., 2010; Jerman, Trebše, \& Mozetič Vodopivec, 2010; Khan, Abert-Vian, Fabiano-Tixier, Dangles, \& Chemat, 2010; Wang, J., Sun, Cao, Tian, \& Li, 2008) and polysaccharides (Fu, C., Tian, Li, Cai, \& Du, 2006; Hromádková \& Ebringerová, 2003; Hromádková, Ebringerová, \& Valachovič, 2002; Yuan, T.-Q., Xu, He, \& Sun, 2010; Zhang, Zhang, Cheung, \& Ooi, 2004). Sonication is known to promote the disruption of plant cell walls, facilitating the release of components towards the extraction media. However, under certain conditions, sonication can also lead to depolymerisation of certain compounds into fragments of lower molar mass (Hromádková et al., 2002) and thus, the effect of this process in the extraction of agar should be carefully investigated.

In this work, the possibility of replacing a more conventional hot water extraction process with a significantly shorter extraction protocol consisting of the combination of hot water and sonication has been evaluated. Furthermore, the effect of eliminating the alkaline purification step to produce non-purified agar fractions which may contain bioactive compounds was also investigated. The composition, structure and antioxidant capacity of 
the generated agar-based extracts, as well as the mechanical performance of the gels produced from these extracts, were characterized, giving the basis for the selection of the optimum extraction protocol in terms of economic and environmental impact, as well as in terms of agar-based extract properties, depending on the final application.

\section{Materials and methods}

\subsection{Materials}

The seaweed Gelidium sesquipedale and the commercial grade agar PRONAGAR were kindly donated by Hispanagar (Burgos, Spain). The dry seaweed was ground into powder before further processing.

Sodium hydroxide, hydrochloric acid (37\%), sodium carbonate, gallic acid (97.5-102.5\%), sulphuric acid $(\geq 97.5 \%)$, trichloroacetic acid $(\geq 99 \%)$, barium chloride $(99 \%)$, porcine gelatine, potassium persulphate $(\geq 99 \%)$, potassium sulphate, phosphate buffered saline tablets, ABTS ( $\geq 98 \%)$ and 6-hydroxy-2,5,7,8-tetramethylchroman-2-carboxylic acid (97\%) were obtained from Sigma-Aldrich (Spain). The Folin-Ciocalteau reagent, modified Lowry reagent and bovine serum albumin were obtained from the "modified Lowry protein assay kit" purchased from Thermo Fisher scientific (Spain).

\subsection{Production of agar-based extracts}

Agar was extracted from raw Gelidium sesquipedale by applying hot water or combined ultrasound-assisted hot water extraction procedures. Additionally, the application of a purification pre-treatment prior to the extraction process was also evaluated.

For the hot water extraction, $50 \mathrm{~g}$ of dry seaweed powder were immersed in $500 \mathrm{~mL}$ of distilled water and heated up to $90^{\circ} \mathrm{C}$ for 2 hours. After that, the agar-based solution was separated from the solid residue by filtration with muslin cloth. The filtrate was allowed to 
gel and it was subsequently frozen overnight at $-21^{\circ} \mathrm{C}$. Then, the material was subjected to two freeze-thaw cycles $\left(-21^{\circ} \mathrm{C} / 25^{\circ} \mathrm{C}\right)$ to improve the elastic properties of the gels and the obtained gel was freeze-dried. The agar-based extract obtained by this method is coded as HW.

For the combined heating-sonication treatment, $50 \mathrm{~g}$ of dry seaweed powder were immersed in $500 \mathrm{~mL}$ of distilled water and placed on a heating plate to reach $90^{\circ} \mathrm{C}$. Once the temperature was stable, an ultrasound probe UP-400S (Hielcher GmbH, Germany) was immersed and a sonication treatment was applied at a maximum power of $400 \mathrm{~W}$ and a constant frequency of $24 \mathrm{kHz}$ for 30 minutes. The agar-based material was separated by filtration, following the same procedure described for the hot water treatment and then, subjected to two freeze-thaw cycles followed by freeze-drying. The agar-based extract obtained by this method is coded as HW-US.

Additionally, a pre-treatment step was also evaluated for both abovementioned treatments. The pre-treatment consisted on soaking $50 \mathrm{~g}$ of dry seaweed powder in $500 \mathrm{~mL}$ of $10 \%(\mathrm{w} / \mathrm{v})$ (2.5M) $\mathrm{NaOH}$ solution and heating up to $90^{\circ} \mathrm{C}$ for 2 hours. After that, the solid material was filtered and washed repeatedly with distilled water using a muslin cloth, until the $\mathrm{pH}$ of the filtrate became neutral. The obtained solid was then subjected to the hot water or combined hot water plus sonication treatments described above, hence obtaining the samples coded as $\mathrm{NaOH}+\mathrm{HW}$ and $\mathrm{NaOH}+\mathrm{HW}-\mathrm{US}$, respectively.

\subsection{Preparation of gels from the agar-based extracts}

Gels were prepared from $1.5 \%(\mathrm{w} / \mathrm{w})$ solutions of the agar-based extracts in distilled water. To dissolve the extracts, the required amount of freeze-dried sample was added to distilled water and heated up to ca. $95^{\circ} \mathrm{C}$ for 30 minutes. Subsequently, the required amount of solution was transferred to glass containers $(2.8 \mathrm{~cm}$ diameter and $4.2 \mathrm{~cm}$ height $)$ for the 
penetration tests. The containers were sealed with aluminium foil and parafilm to avoid water evaporation and they were left to equilibrate at room temperature overnight.

\subsection{Chemical composition of agar-based extracts}

\subsubsection{Carbohydrate content}

The total carbohydrate and galactose content in the extracts was determined after sulphuric acid hydrolysis, following the method described in (Martínez-Abad, Giummarella, Lawoko, \& Vilaplana, 2018). A two-step sulphuric acid hydrolysis was performed by incubating 4 mg of sample with $250 \mu \mathrm{L}$ of $72 \% \mathrm{H}_{2} \mathrm{SO}_{4}$ at room temperature for $3 \mathrm{~h}$; the solution was diluted with $1250 \mu \mathrm{L}$ of deionized water and subsequently incubated at $100{ }^{\circ} \mathrm{C}$ for 3 hours. The samples were then analyzed using high performance anion exchange chromatography with pulsed amperometric detection (HPAEC-PAD) with a 940 IC system (Metrohm) equipped with a Matrosep Carb 2 column $(4 \times 250 \mathrm{~mm}$, Metrohm). Control samples of known concentrations of mixtures of glucose, fucose, galactose, arabinose, xylose, mannose, galacturonic acid or glucuronic were used for calibration. All experiments were carried out in triplicate.

\subsubsection{Protein content}

Total protein content was measured following the Lowry method (Lowry, Rosebrough, Farr, \& Randall, 1951) with some minor modifications. Briefly, $1 \mathrm{~mL}$ of the modified Lowry reagent was mixed with $0.2 \mathrm{~mL}$ of the agar-based extracts (dissolved in distilled water at a concentration of $5 \mathrm{mg} / \mathrm{mL}$ ) and incubated for 10 minutes at room temperature. Then, $0.1 \mathrm{~mL}$ of Folin-Ciocalteau reagent (previously diluted 1:1 with distilled water) were added and vortexed, incubating the resulting solution for 30 minutes at room temperature. The absorbance values were read at $750 \mathrm{~nm}$. A calibration curve was prepared with serial 
dilutions of bovine serum albumin (BSA) and the total protein content was expressed as $\mathrm{mg}$ BSA/g extract. All determinations were carried out in triplicate.

\subsubsection{Phenolic content}

Total phenolic content was estimated by the Folin-Ciocalteau colorimetric assay (Singleton, Orthofer, \& Lamuela-Raventós, 1999). Briefly, Folin-Ciocalteau reagent was diluted 1:10 with distilled water and $1 \mathrm{~mL}$ of the final dilution was mixed with $0.2 \mathrm{~mL}$ of the extract sample (dissolved in distilled water at a concentration of $5 \mathrm{mg} / \mathrm{mL}$ ) at room temperature. Finally, $0.8 \mathrm{~mL}$ of sodium carbonate $(75 \mathrm{mg} / \mathrm{mL})$ were added and the samples were heated up to $50^{\circ} \mathrm{C}$ during 30 minutes. Absorbance values were read at $750 \mathrm{~nm}$. A calibration curve was built using gallic acid as the standard, and the total phenolic content was expressed as mg of gallic acid (GA)/g extract. All determinations were carried out in triplicate.

\subsubsection{Sulphate content}

Sulphate content was measured turbidimetrically using $\mathrm{BaCl}_{2}$-gelatin method after hydrolysis in $0.5 \mathrm{M} \mathrm{HCl}$ as described previously (Dodgson \& Price, 1962) with slight modifications. Agar-based extracts $(5 \mathrm{mg})$ were hydrolysed in $1 \mathrm{~mL}$ of $1 \mathrm{M} \mathrm{HCl}$ at $100{ }^{\circ} \mathrm{C}$ for 5 hours. After cooling, the solutions were mixed thoroughly and filtered with a Whatman No. 1 filter paper. A portion $(0.2 \mathrm{~mL})$ was transferred to a $10 \mathrm{~mL}$ tube containing $3.8 \mathrm{~mL}$ of $3 \%$ trichloroacetic acid and $1 \mathrm{~mL}$ of $\mathrm{BaCl}_{2}$-gelatin reagent. The solution was mixed vigorously and incubated at room temperature for 15 minutes. The absorbance at $360 \mathrm{~nm}$ was measured. A blank was prepared in the same manner, except that distilled water was used instead of the agar sample. A calibration curve was prepared using $\mathrm{K}_{2} \mathrm{SO}_{4}$ at concentration ranging from 0.06 to $0.6 \mathrm{mg} / \mathrm{mL}\left(\sim 0.0533-0.533 \mathrm{mg} \mathrm{SO}_{4}{ }^{2-} / \mathrm{mL}\right)$. The sulphate 
content was calculated and expressed as a percentage on a dry weight basis. All determinations were carried out in triplicate.

\section{5 $\mathrm{ABTS}^{\cdot+}$ radical cation scavenging activity}

The ABTS $^{++}$radical cation scavenging activity of the different agar-based extracts was determined according to (Re et al., 1999). Briefly, $0.192 \mathrm{~g}$ of ABTS were dissolved in 50 $\mathrm{mL}$ of PBS at $\mathrm{pH} 7.4$ and mixed with $0.033 \mathrm{~g}$ of potassium persulfate overnight in the dark to yield the $\mathrm{ABTS}^{++}$radical cation. Prior to use in the assay, the ABTS ${ }^{++}$was diluted with PBS for an initial absorbance of $\sim 0.700 \pm 0.02$ (1:50 ratio) at $734 \mathrm{~nm}$, at room temperature. Free radical scavenging activity was assessed by mixing $1.0 \mathrm{~mL}$ diluted ABTS ${ }^{+}$with $10 \mu \mathrm{L}$ of aqueous solutions of the agar-based extracts and monitoring the change in absorbance at $0,1,5,10,15$ and 30 minutes (until a steady state was achieved). A calibration curve was developed by using 6-hydroxy-2,5,7,8-tetramethylchromane-2-carboxylic acid (Trolox). The antioxidant capacity of test extracts was expressed as mg Trolox equivalents (TE)/g extract. All determinations were carried out in triplicate.

\subsection{Fourier transform infrared spectroscopy (FT-IR)}

Freeze-dried agar-based samples were analyzed by FT-IR in attenuated total reflectance (ATR) mode using a Thermo Nicolet Nexus (GMI, USA) equipment. The spectra were recorded at $4 \mathrm{~cm}^{-1}$ resolution in a wavelength range between $400-4000 \mathrm{~cm}^{-1}$ and averaging a minimum of 32 scans.

\subsection{UV-Vis spectrophotometry}

Aqueous solutions of the agar-based extracts $(0.5 \mathrm{mg} / \mathrm{mL})$ were prepared by dissolving the required amount of material in distilled water and heating up to $95^{\circ} \mathrm{C}$ for 30 minutes. The 
samples were kept warm until the measurements were performed. The UV-Vis spectra in the wavelength range of 200-1000 $\mathrm{nm}$ were recorded using a 8453 Agilent UV-Vis spectrophotometer.

\subsection{High performance size exclusion chromatography (HPSEC)}

The molecular weight of the agar samples was estimated by HPSEC analyses. These were performed using a high performance liquid chromatography system equipped with a Waters 2695 separations module, a Waters 2414 refractive index detector and a Waters 2487 dual UV/visible detector (Waters, USA). The samples $(1 \mathrm{mg} / \mathrm{mL})$ were dissolved in the mobile phase $\left(0.05 \mathrm{M} \mathrm{Na}_{2} \mathrm{SO}_{4} / 0.01 \mathrm{M}\right.$ EDTA, $\left.\mathrm{pH} 7\right)$ at $97{ }^{\circ} \mathrm{C}$ for $1 \mathrm{~h}$, filtered through $0.8 \mu \mathrm{m}$ pore syringe filters for aqueous media (Sartorius, Germany) and injected into an OHpak SB-806 HQ (8 mm x $300 \mathrm{~mm}$ ) size exclusion chromatography column (Shodex, Japan) equilibrated at $40{ }^{\circ} \mathrm{C}$. The injection volume was $20 \mu \mathrm{L}$ and the flow rate was $0.5 \mathrm{~mL} / \mathrm{min}$. Calibration was performed using P-82 pullulan standards (Shodex, Japan), and peak molecular weights $\left(\mathrm{M}_{\mathrm{p}}\right)$ are reported.

\subsection{X-ray diffraction (XRD)}

XRD measurements of the freeze-dried agar-based extracts were carried out on a D5005 Bruker diffractometer. The instrument was equipped with a $\mathrm{Cu}$ tube and a secondary monochromator. The configuration of the equipment was $\theta-2 \theta$, and the samples were examined over the angular range of $3^{\circ}-60^{\circ}$ with a step size of $0.02^{\circ}$ and a count time of 200 s per step. Peak fitting was carried out using the Igor software package (Wavemetrics, Lake Oswego, Oregon), similarly to the procedure described in a previous work (Martínez-Sanz, Lopez-Sanchez, Gidley, \& Gilbert, 2015). The obtained values from the fitting coefficients are those that minimize the value of Chi-squared, which is defined as: 
$\chi^{2}=\Sigma\left(\frac{y-y_{i}}{\sigma_{i}}\right)^{2}$

where $\mathrm{y}$ is a fitted value for a given point, $y_{i}$ is the measured data value for the point and $\sigma_{\bar{i}}$ is an estimate of the standard deviation for $y_{i}$. The curve fitting operation is carried out iteratively and for each iteration, the fitting coefficients are refined to minimize $\chi^{2}$. The crystallinity index $\mathrm{X}_{\mathrm{C}}$ was determined from the obtained fitting results by applying the following equation:

$$
X_{C}(\%)=\frac{\sum A_{\text {Crystal }}}{A_{\text {Total }}} \times 100
$$

where $A_{\text {Total }}$ is the sum of the areas under all the diffraction peaks and $\Sigma A_{\text {Crystal }}$ is the sum of the areas corresponding to the crystalline peaks.

\subsection{Thermogravimetric analyses (TGA)}

Thermogravimetric curves (TG) were recorded with a Setaram Setsys 16/18 (SETARAM Instrumentation, France). The samples (ca. $10 \mathrm{mg}$ ) were heated from 30 to $1000^{\circ} \mathrm{C}$ with a heating rate of $10^{\circ} \mathrm{C} / \mathrm{min}$ under nitrogen atmosphere. Derivative TG curves (DTG) express the weight loss rate as a function of temperature.

\subsection{Penetration tests}

Penetration test was used to obtain gel fracture force and determine gel strength. Measurements were performed at room temperature $\left(25-30{ }^{\circ} \mathrm{C}\right)$ using a texture analyzer (Stable Micro Systems model TA-XT2, Surrey, UK) equipped with a $1 \mathrm{~cm}^{2}$ cylindrical Teflon plunger and operating at a penetration rate of $0.2 \mathrm{~mm} / \mathrm{s}$ to a depth of $5 \mathrm{~mm}$. All measurements were performed, at least, in triplicate. 


\subsection{Statistical analysis}

Data analysis was carried out using Statgraphics Stratus by Statgraphics Technologies, Inc. One-way analysis of variance (ANOVA) was done to determine the significant differences between sample means, at a significance level of $\mathrm{P}<0.05$. Mean comparisons were performed by the LSD (for the antifungal activity tests) or the Tukey Test (for the composition analysis).

\section{Results and Discussion}

\subsection{Extraction yield and composition of the agar-based extracts}

Different extraction processes were utilized to extract agar from Gelidium sesquipedale and the structure and properties of the obtained materials were characterized. In the first place, the feasibility of reducing the extraction time (by 4-fold) by replacing the conventional hot water extraction with a shorter treatment combining heating with sonication was evaluated. Furthermore, the effect of an initial alkaline pre-treatment step to remove impurities from the raw seaweed, prior to the conventional and the sonication-heat combined extraction processes, was also investigated. The extraction yields were $11.9 \pm 1.4 \%, 10.1 \pm 0.1 \%, 2.9$ $\pm 0.8 \%$ and $2.3 \pm 0.3 \%$ for the HW, HW-US, $\mathrm{NaOH}+\mathrm{HW}$ and $\mathrm{NaOH}+\mathrm{HW}-\mathrm{US}$ extracts, respectively. Comparison of these extraction yields with values previously reported in the literature is extremely complex due to (i) the large variability in the extraction parameters (such as extraction time, extraction temperature and water-to-seaweed ratio) reported in the literature and (ii) the variability in the agar content in the different seaweed species used. Amongst all the extraction parameters, the extraction time has been previously reported as one of the main factors affecting the agar extraction yield and maximum yields have been attained with extraction times ranging from $2 \mathrm{~h}$ to $4 \mathrm{~h}$ (Arvizu-Higuera et al., 2008; Kumar $\&$ Fotedar, 2009). The highest extraction yields reported for seaweed from the family of 
Gelidiaceae (ca. 30\%) correspond to the agars from Gelidium serrulatum and Gelidium floridanum, obtained by performing 3 h extractions (Lemus, Bird, Kapraun, \& Koehn, 1991). Different seaweed species present very variable extraction yields. For instance, for extraction times close to $2 \mathrm{~h}$, a yield of ca. $13 \%$ was reported for Gracialaria manilaensis species (Ahmad et al., 2011), whereas higher yields of ca. $27 \%$ and $55 \%$ have been reported for Gracilaria corticata (Yousefi, Islami, \& Filizadeh, 2013) and Gracilaria cliftonii (Kumar \& Fotedar, 2009), respectively. Thus, it is evident that the agar extraction parameters should be optimized individually for each particular seaweed species. Future work will be carried out to determine the optimum extraction parameters required to maximize the extraction yield and the agar properties from the Gelidium sesquipedale species used in this work. It is interesting to note that the combination of the hot water treatment with sonication allowed reducing 4-fold the extraction time while only a slight decrease in the yield was obtained (the difference not being statistically significant, $\mathrm{p} \leq 0.05$ ). This evidences the ability of sonication in promoting the extraction of compounds from the raw seaweed and shows the great potential of the combined sonication-heat treatment to generate agar-based extracts by a more energy efficient method. Nonetheless, the extraction parameters such as time, temperature and water-to-seaweed ratio will have to be optimized for this particular case to fully exploit this alternative extraction approach.

One fact that is consistently reported in the literature is that alkali pre-treatment results in significantly decreased extraction yields (Arvizu-Higuera et al., 2008; Freile-Pelegrín, Y \& Robledo, 1997; Kumar \& Fotedar, 2009; Rath \& Adhikary, 2004). Alkali treatment is known to convert L-galactose-6-sulphate to 3,6-anhydro-L-galactose, hence improving the gel forming ability of the agar and leading to increased gel strength (Freile-Pelegrın \& Murano, 2005). On the other hand, the agar undergoes degradation and diffusion towards the aqueous 
medium during the alkali treatment, thus significantly reducing the extraction yield (Ahmad et al., 2011; Kumar \& Fotedar, 2009). As evidenced by the marked decrease in the extraction yields, the alkali pre-treatment prior to the hot water extraction may have been too harsh for the Gelidium sesquipedale species. Thus, the alkali concentration, pre-treatment time and temperature also need to be optimized to minimize agar loses and enhance the extraction yield, but it is useful in this work for comparative purposes.

These different applied extraction protocols were expected to result in differences in the composition of the agar-based extracts, which will consequently determine the structure and properties of these materials. Prior to their structural characterization, the ATR-FTIR spectra from the agar-based extracts were recorded to identify major compositional differences. As observed in Figure 1, all the samples presented the most characteristic bands from agar (pointed out by black arrows in Figure 1B), located at $930 \mathrm{~cm}^{-1}$, assigned to the presence of 3,6-anhydro-galactose residue, and at $890 \mathrm{~cm}^{-1}$, corresponding to the $\mathrm{C}-\mathrm{H}$ bending at the anomeric carbon in $\beta$-galactopyranosyl residues (Gómez-Ordóñez \& Rupérez, 2011; Pereira, Sousa, Coelho, Amado, \& Ribeiro-Claro, 2003). The relative intensity of these bands was stronger for the materials obtained after applying the alkaline pre-treatment, suggesting that, as expected, a higher degree of purity was attained. An important consideration for the quality of agar-based materials is their degree of sulphation. The presence of sulphate ester groups in the galactose units of agar samples is detrimental for their gelling behaviour, i.e. weaker gels are formed (Freile-Pelegrín, Y \& Robledo, 1997; Murano, 1995). Two bands related to the degree of sulphation in agars, pointed out by grey arrows in Figure 1B, were those appearing at $1260 \mathrm{~cm}^{-1}$, characteristic of sulphate ester groups (Gómez-Ordóñez \& Rupérez, 2011), and at $1150 \mathrm{~cm}^{-1}$, ascribed to the vibration mode of ester-sulphate linkages (Guerrero et al., 2014). As observed, these bands were visible in all the samples, confirming 
that all of them possesed sulphate groups. Furthermore, the presence of shoulders located at $840-850 \mathrm{~cm}^{-1}$ and ca. $820 \mathrm{~cm}^{-1}$ suggests the existence of sulphate groups at the C-4 position in the D-galactose units (Gómez-Ordóñez \& Rupérez, 2011; Prado-Fernández, RodríguezVázquez, Tojo, \& Andrade, 2003) and at the C-6 of L-galactose units (Mazumder et al., 2002; Souza et al., 2012), respectively. It is worth noting that the relative intensity of the band characteristic of the 3,6-anhydro-galactose residue to that of the 6-sulphate on the Lgalactose unit was significantly higher for the $\mathrm{NaOH}+\mathrm{HW}$ and $\mathrm{NaOH}+\mathrm{HW}$-US extracts as compared with the HW and HW-US samples. This confirms that, as suggested in previous studies (Mazumder et al., 2002; Souza et al., 2012), alkali treatment leads to the conversion of L-galactose 6-sulphate to 3,6-anhydrogalactose. Although most of the agar characteristic bands were located at approximately the same wavenumber, differences in relative intensities were evident, especially when comparing the non-purified agar extracts (HW and HW-US) to the purified ones (the commercial, $\mathrm{NaOH}+\mathrm{HW}$ and $\mathrm{NaOH}+\mathrm{HW}-\mathrm{US}$ samples). It is worth noting that while the purified agars showed two bands located at ca. $1360 \mathrm{~cm}^{-1}$ and $1430 \mathrm{~cm}^{-1}$, the non-purified extracts presented a broader band centred at ca. $1400 \mathrm{~cm}^{-1}$. Furthermore, the small band at $1298 \mathrm{~cm}^{-1}$ shifted to $1317 \mathrm{~cm}^{-1}$ in the non-purified agars. All these bands arise from the bending and stretching of $\mathrm{C}-\mathrm{H}$ and $\mathrm{C}-\mathrm{O}$ bonds in polysaccharides (Yuan, T.-Q. et al., 2010) and, thus, the differences between samples are indicative of the presence of impurities in the non-purified agars. Furthermore, although the amide I band, typically located at $1653 \mathrm{~cm}^{-1}$, was masked by the intense band corresponding to bound water (located at ca. $1630 \mathrm{~cm}^{-1}$ ), the amide II band, located at $1540 \mathrm{~cm}^{-1}$, was detected for all the samples, evidencing the presence of a significant amount of protein in the extracts. The raw Gelidium sesquipedale has been shown to contain ca. $30 \%$ of protein in weight (Faraj, Lebbar, Debry, \& Najim, 1987) and, consequently, it is not surprising that a certain amount of these proteins was extracted with the applied treatments. In particular, a greater 
protein content is expected in the case of the non-purified extracts, as deduced from the shape of the $\mathrm{OH}$ and $\mathrm{NH}$ stretching bands (and demonstrated below).
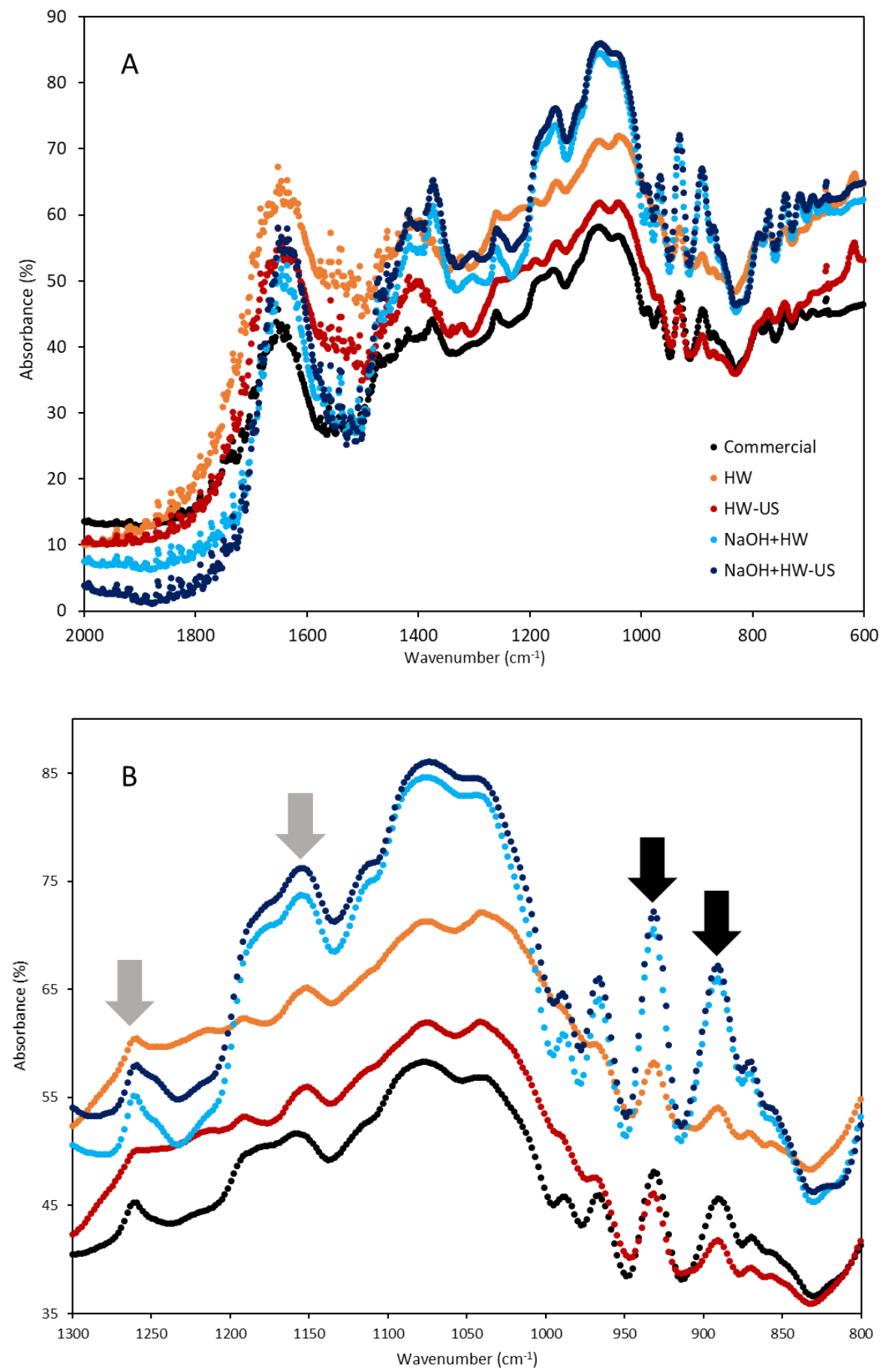

Figure 1. ATR-FTIR spectra from the agars extracted from Gelidium sesquipedale. Magnification within the $2000-600 \mathrm{~cm}^{-1}$ region (A) and $1300-800 \mathrm{~cm}^{-1}$ (B). 
Quantification of different components was carried out to determine the composition of the agar-based extracts depending on the extraction protocol and the results are compiled in Table 1. The first clear observation is that the alkali pre-treated samples, along with the commercial agar, presented the highest polysaccharide contents, with very little amounts of proteins and polyphenols. Additionally, more than $95 \%$ of their carbohydrate content could be ascribed to galactose, indicating the selectivity of the process in extracting agars with a relatively high purity. On the other hand, the HW and HW-US extracts presented lower carbohydrate contents, with a lower contribution of galactose (around $70 \%$ of the total carbohydrate content), pointing towards the presence of other hemicellulosic components in the extracts. These extracts contained, instead, significantly greater amounts of proteins and polyphenols. It is also worth noting that the combination of the hot water treatment with sonication promoted the extraction of polyphenols and, especially, proteins, without affecting the amount of extracted polysaccharides, even though the extraction time was reduced 4-fold with respect to the hot water treatment. Thus, the combination of hot water with sonication may be interesting for the production of multi-component extracts, which could show interesting functional properties as described below.

A complete mass balance could not be achieved as ashes present in the extracts, as evidenced by XRD analyses (cf. Figure 2) which usually constitute 10-25 wt.-\% in these type of algae (Alvarez-Gómez, Korbee, \& Figueroa, 2016; Foster \& Hodgson, 1998; Marinho-Soriano, Fonseca, Carneiro, \& Moreira, 2006), or lipids (typically accounting for less than 1 wt.-\%) (Foster \& Hodgson, 1998; Marinho-Soriano et al., 2006) were not quantified. To the best of our knowledge, there are no works available on the literature reporting on the composition of the agar-based materials extracted from Gelidium sesquipedale seaweed. The hot-water extract from Gelidium amansii, produced similarly to HW in this work, has been reported to 
contain $67 \%$ of sugars, with the main components being galactose $(55.1 \%)$, mannose $(5.7 \%)$, xylose (6.2\%) and fucose (3.9\%) (Fu, Y.-W., Hou, Yeh, Li, \& Chen, 2007). Our results show that agar extracts with either higher purity, as in the case of those extracts generated with the alkali pre-treatment, or with more heterogeneity and potential interesting properties may be obtained by tuning the extraction conditions.

As previously mentioned, the amount of sulphate groups in agar-based materials is an important attribute since it is related to the ability of the agar to form strong gels. As deduced from Table 1 , the purified extracts $(\mathrm{NaOH}+\mathrm{HW}$ and $\mathrm{NaOH}+\mathrm{HW}-\mathrm{US})$ and the commercial agar presented very similar sulphate contents, which are close to those reported in the literature for the agar extracted with boiling water from Gracilaria secundata $(2.1 \%)$ (Lignell \& Pedersén, 1989), Gelidium serrulatum and Gelidium floridanum (2.4-2.8\%) (Lemus et al., 1991), but slightly higher than that of agar extracted from Gelidium sesquipedale harvested in Morocco (1.0-1.6\%) (Mouradi-Givernaud et al., 1999). The nonpurified extracts (HW and HW-US) presented greater sulphate contents, even though the amount of polysaccharides in those samples were lower than in the purified extracts, indicating a much higher ratio of agaropectin.

Many components such as sulphated polysaccharides and polyphenols from red seaweed have been reported to present antioxidant properties (Jiménez-Escrig, Jiménez-Jiménez, Pulido, \& Saura-Calixto, 2001; Rocha de Souza et al., 2007; Wijesekara, Pangestuti, \& Kim, 2011; Yuan, Y. V. \& Walsh, 2006). Therefore, the antioxidant capacity of the agar-based extracts from Gelidium sesquipedale was evaluated throught the ABTS method and correlated to the different composition of the extracts. As deduced from Table 1, the antioxidant capacity was significantly higher for the HW and HW-US extracts. This was 
already anticipated due to the higher polyphenol and protein content of these extracts. The estimated antioxidant capacities (ca. 72 and $71 \mu \mathrm{mol}$ TE/g extract for the HW and HW-US extracts, respectively) were much higher than those previously reported for the water extracts from Gelidium pusillum and Gelidium corneum seaweed (lower than $4 \mu \mathrm{mol}$ TE/g extract), which seems to be related to the lower phenolic content of these two species (ca. 6.5 and 3.5 mg GA/g sample) (Alvarez-Gómez et al., 2016). In fact, a lower antioxidant capacity of 39.4 $\mu \mathrm{mol} \mathrm{TE} / \mathrm{g}$ extract has also been reported for the organic extract (produced using $70 \%$ methanol) from Geldium amansii seaweed, which presented a lower polyphenol content of ca. $14.1 \mathrm{mg} \mathrm{GA} / \mathrm{g}$ sample (Lee, J.-H. \& Kim, 2015). Very few works have reported on the antioxidant capacity of water-soluble extracts from red seaweed; instead, organic solvents such as hexane, chloroform and methanol are preferred to produce extracts with antioxidant capacity, since bioactive compounds such as non-glycosilated flavonoids, phospholipids, carotenoids and chlorophyll analogues, are known to be more soluble in these solvents (Nakayama, Tamura, Kikuzaki, \& Nakatani, 1999). However, other bioactive substances such as sulphated polysaccharides and glycosilated polyphenols are expected to be more easily extracted with water. The antioxidant acitvity of sulphated polysaccharides from red seaweed, such as carrageenans, has been suggested to be positively correlated with their sulphate content (Rocha de Souza et al., 2007). Although the HW and HW-US extracts presented greater sulphate contents than the commercial agar and the alkali-treated extracts, the main compositional difference laid in their protein content. Some studies have demonstrated that apart from polyphenols, hydrophilic peptides and aminoacids from red seaweed may be resposible for the antioxidant capacity of water-soluble extracts (Wang, T. et al., 2010; Yuan, Y. V., Westcott, Hu, \& Kitts, 2009). Thus, the increased antioxidant capacity of the HW and HW-US extracts is most likely due to the presence of impurities such as peptides, aminoacids and glycosilated polyphenols. 
It should be noted that the HW and HW-US extracts also showed higher antioxidant capacity than the water extracts from several red and green seaweed species, such as Porphyra umbilicalis, Halopythis incurva, Gracilariopsis longissima, Hydropuntia cornea, Ulva rotundata and Lichina pygmaea (Alvarez-Gómez et al., 2016), which were extracted at 45 ${ }^{\circ} \mathrm{C}$ for $6 \mathrm{~h}$. Thus, the non-purified HW and HW-US agar-based extracts show a great potential to be used as bioactive ingredients due to their relatively high antioxidant capacity.

Table 1. Composition and antioxidant capacity of the agar-based extracts synthesised from Gelidium sesquipedale. Data shown as mean $+/-\mathrm{SD}, \mathrm{n}=3$.

\begin{tabular}{|l|c|c|c|c|c|c|}
\cline { 2 - 7 } \multicolumn{1}{l|}{} & $\begin{array}{c}\text { Carbohydrate } \\
\text { content } \\
(\mathrm{mg} / \mathrm{g} \text { sample })\end{array}$ & $\begin{array}{c}\text { Galactose } \\
\text { content }^{\mathrm{a}} \\
(\mathrm{mg} / \mathrm{g} \text { sample })\end{array}$ & $\begin{array}{c}\text { Protein } \\
(\mathrm{mg} \mathrm{BSA} / \mathrm{g} \\
\text { sample })\end{array}$ & $\begin{array}{c}\text { Polyphenols } \\
(\mathrm{mg} \mathrm{GA} / \mathrm{g} \\
\text { sample })\end{array}$ & $\begin{array}{c}\text { Sulphate } \\
(\%)\end{array}$ & $\begin{array}{c}\text { Antioxidant } \\
\text { Capacity } \\
\text { ABTS } \\
(\mathrm{mg} \mathrm{TE} / \mathrm{g} \\
\text { extract })\end{array}$ \\
\hline Commercial & $683 \pm 14^{\mathrm{a}}$ & $648 \pm 9^{\mathrm{a}}$ & $23 \pm 7^{\mathrm{a}}$ & $6.9 \pm 0.1^{\mathrm{b}}$ & $2.0 \pm 0.2^{\mathrm{a}}$ & $9 \pm 2^{\mathrm{a}}$ \\
\hline $\mathrm{HW}$ & $392 \pm 77^{\mathrm{b}}$ & $291 \pm 56^{\mathrm{b}}$ & $136 \pm 9^{\mathrm{b}}$ & $30 \pm 2^{\mathrm{c}}$ & $2.8 \pm 0.2^{\mathrm{b}}$ & $18 \pm 4^{\mathrm{b}}$ \\
\hline $\mathrm{HW}-\mathrm{US}$ & $325 \pm 32^{\mathrm{b}}$ & $237 \pm 23^{\mathrm{b}}$ & $251 \pm 12^{\mathrm{c}}$ & $47 \pm 1^{\mathrm{d}}$ & $3.1 \pm 0.1^{\mathrm{b}}$ & $18 \pm 3^{\mathrm{b}}$ \\
\hline $\begin{array}{l}\mathrm{NaOH}+\mathrm{HW} \\
-\mathrm{US}\end{array}$ & $800 \pm 122^{\mathrm{a}}$ & $728 \pm 108^{\mathrm{a}}$ & $16 \pm 3^{\mathrm{a}}$ & $3.2 \pm 0.2^{\mathrm{a}}$ & $1.7 \pm 0.1^{\mathrm{a}}$ & $8 \pm 3^{\mathrm{a}}$ \\
\hline
\end{tabular}

${ }^{\text {a }}$ Quantification of galactose includes both galactosyl and 3,6-dehydrogalactosyl units in agar.

Values with different letters are significantly different $(\mathrm{p} \leq 0.05)$.

\subsection{Structural characterization of the agar-based extracts}

The effect of the different extraction protocols in the structure of the obtained agars was also investigated. The crystalline structure of the seaweed and the agar-based extracts was 
investigated by means of XRD characterization and the obtained patterns are shown in Figure 2. The raw Gelidium sesquipedale presented a complex diffraction pattern with multiple peaks and a calculated crystallinity index of ca. $23.1 \%$. Some of these peaks may arise from the presence of mineral compounds, such as $\underline{\text { silica }}\left(\mathrm{SiO}_{2}\right)$ and weddellite $\left(\mathrm{CaC}_{2} \mathrm{O}_{4} \cdot 2 \mathrm{H}_{2} \mathrm{O}\right)$, that have been previously detected in the XRD spectra from Gelidium seaweed (Chen, Y. W., Lee, Juan, \& Phang, 2016; Singh, Gaikwad, Park, \& Lee, 2017). These peaks were visible in the spectra from the HW and HW-US extracts, but were absent in the purified extracts, confirming the removal of impurities and isolation of agar in these samples. The commercial agar presented a pattern similar to that previously shown for pure agar films, with one well-defined peak at $19.0^{\circ}$ and a shoulder at $13.9^{\circ}$ (Atef et al., 2014; Freile-Pelegrín, Y. et al., 2007; Guerrero et al., 2014). This suggests that the agar presented a certain degree of order in its structure, as opposed to the patterns typically reported for amorphous agar samples, which only present a diffuse broad band at $20^{\circ}$ (Guerrero et al., 2014). As deduced from the estimated crystallinity values, shown in Figure 3 , the $\mathrm{NaOH}+\mathrm{HW}$ and $\mathrm{NaOH}+\mathrm{HW}-\mathrm{US}$ extracts presented a more crystalline structure than the commercial agar, which may be due to a different alkaline pre-treatment being applied in the case of the commercial sample. It is possible that the alkaline treatment applied in this work, besides removing impurities from the raw seaweed, may have resulted in the digestion of the more amorphous agar domains, as already suggested by the low extraction yields attained with the alkali pre-treatment. It should be noted that, although the HW and HW-US extracts presented similar crystallinity values to the commercial agar, only $44 \%$ and $26 \%$ of the crystalline area in these samples corresponded to the peaks assigned to the agar fraction, which is in agreement with the results from the composition analyses (i.e. the carbohydrate content was ca. $40 \%$ in HW and $30 \%$ in HW-US.) 


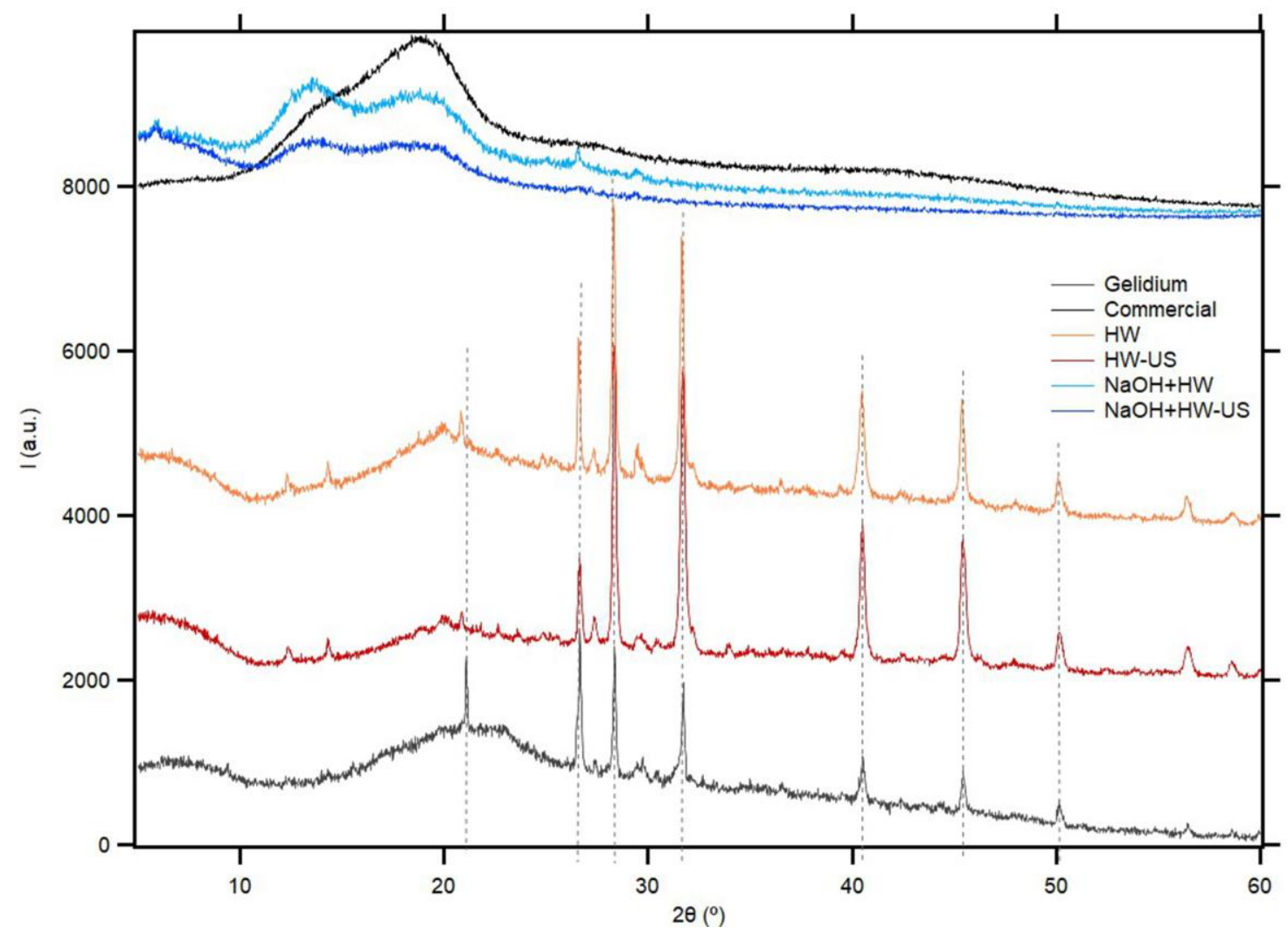

Figure 2. XRD patterns from the raw Gelidium sesquipedale and the agar-based extracts. The patterns corresponding to the commercial, $\mathrm{NaOH}+\mathrm{HW}$ and $\mathrm{NaOH}+\mathrm{HW}-\mathrm{US}$ samples have been offset for clarity.

In addition to the sulphate content, the molecular weight distribution of agar has been seen to play an important role on the gelling properties, with greater gel strength for those samples presenting higher molecular weight and narrower distribution (Sousa-Pinto, Murano, Coelho, Felga, \& Pereira, 1999). The HPSEC chromatograms and peak molecular weight values for the different samples are shown in Figure 3. All chromatograms obtained using the refractive index detector showed a broad band ascribed to the high molecular-weight components of the extract, followed by a narrower band $\left(t_{R}=23.6\right.$ minutes $)$ corresponding to the void volume of the column, where the small molecular weight compounds could not be separated and co-eluted. As observed, all the agar-based extracts presented greater molecular weights than that of the commercial agar. This is likely related to the differences 
in the extraction protocols used to generate the samples. For the agar-based extracts, the alkaline pre-treatment somewhat decreased the molecular weight of the agar fraction, which may be due to a partial digestion of the agar by the alkali medium. Lower molar weights were also obtained when the sonication treatment was applied, as previously reported for the extraction of other polysaccharides from vegetable biomass (Hromádková et al., 2002). Although the extraction protocol and the characteristics of the raw seaweed are determinant for the molecular weight of the extracted agar, as a reference, lower molecular weights of $2.65 \cdot 10^{2} \mathrm{kDa}$ have been reported for the agar extracted from Gelidium pulchellum (SousaPinto et al., 1999).

It is worth noting that, while the absorbance of agar at $280 \mathrm{~nm}$ is negligible, proteins and, especially, polyphenols have a maximum of absorption at around that wavelength due to the presence of aromatic rings in their structure (Gómez-Mascaraque, Dhital, Lopez-Rubio, \& Gidley, 2017; Whitaker \& Granum, 1980), being detectable by the UV-vis detector coupled to the HPSEC equipment. The UV-vis chromatograms of the commercial and the $\mathrm{NaOH}$ pretreated extracts exhibited only a small peak centred at 23.6 minutes, due to their low content of polyphenols (cf. Table 1). In contrast, this band was considerably more intense in the UVvis chromatograms of the HW and HW-US extracts, confirming the presence of higher amounts of low molecular weight impurities in these materials. Interestingly, the latter also showed a broader and less intense band matching the retention times corresponding to the high molecular weight agar molecules, suggesting that part of these impurities were strongly interacting and, therefore, co-eluted with the polysaccharide. It is also worth noting that a shift in the maximum of those bands to lower retention times could be observed in the UVvis chromatograms with respect to the RI chromatograms, suggesting that a greater amount of impurities was bound to the polysaccharide chains with the highest molecular weights. 
Indeed, longer polymeric chains have a greater number of functional groups per molecule, which might involve greater chances for interaction with other molecules.

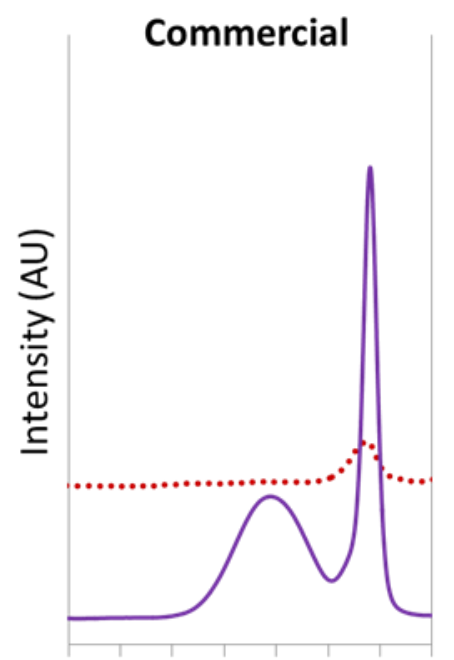

1214161820222426 $\mathrm{R}_{\mathrm{t}}$ (min)

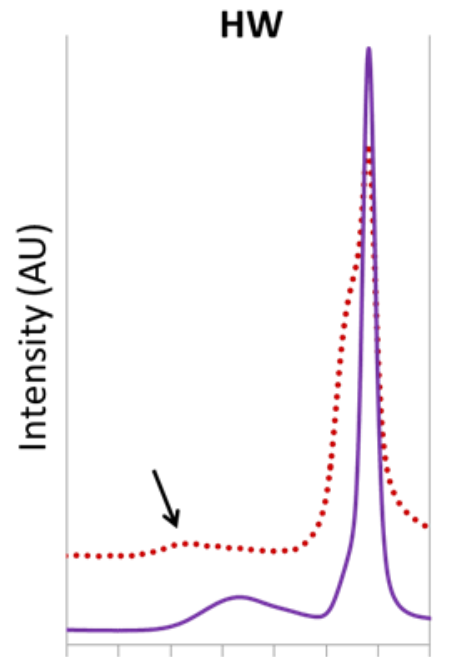

1214161820222426 $\mathrm{R}_{\mathrm{t}}$ (min)

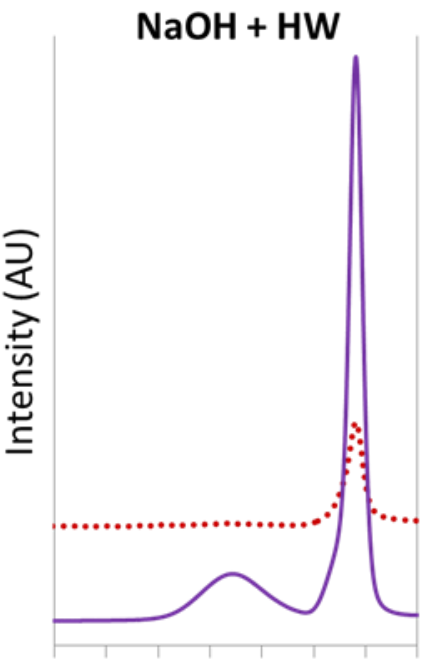

1214161820222426

$\mathrm{R}_{\mathrm{t}}$ (min)

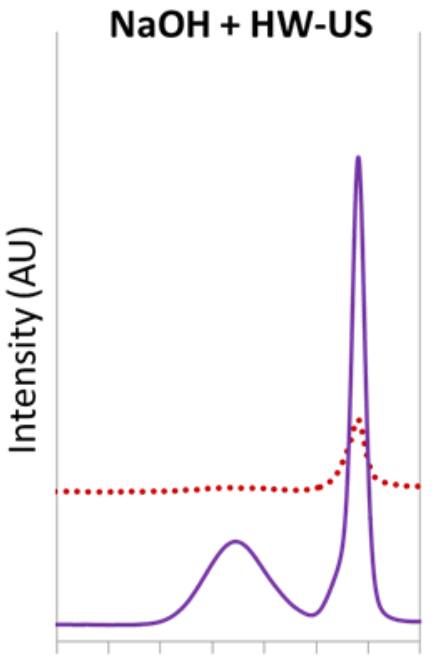

1214161820222426

$R_{t}(\min )$

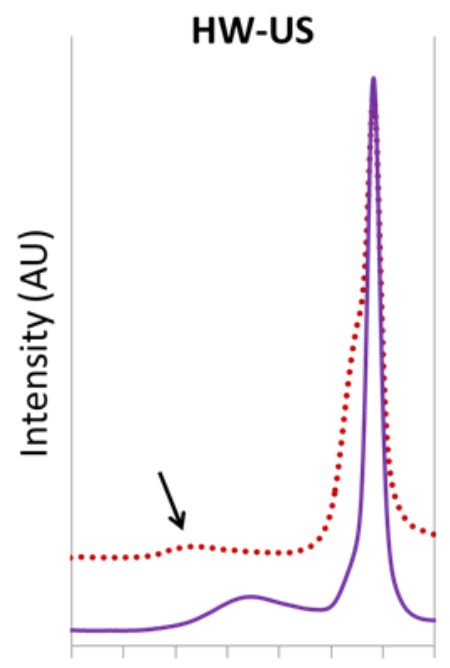

\begin{tabular}{|l|c|c|}
\cline { 2 - 3 } \multicolumn{1}{c|}{} & $\begin{array}{c}M p \cdot 10^{3} \\
\text { (Da) }\end{array}$ & $\begin{array}{c}\mathrm{X}_{\mathrm{C}} \\
(\%)\end{array}$ \\
\hline Commercial & 251 & 17.1 \\
$\mathrm{HW}$ & 1112 & 25.3 \\
$\mathrm{HW}-$ US & 920 & 37.5 \\
$\mathrm{NaOH}+\mathrm{HW}$ & 781 & 21.6 \\
$\mathrm{NaOH}+\mathrm{HW}-\mathrm{US}$ & 569 & 25.5 \\
\hline
\end{tabular}

Figure 3. HPSEC chromatograms obtained from the refractive index (solid lines) and UVvisible (dotted lines) detectors, together with the peak molecular weight and crystallinity (estimated from XRD) of the agar-based extracts synthesised from Gelidium sesquipedale. The retention times $\left(\mathrm{R}_{\mathrm{t}}\right)$ corresponding to the refractive index detector have been corrected to account for the lag time between both detectors. 
TGA analyses were also carried out to determine the effect of the presence of impurities in the thermal stability of the extracts. As shown in Figure 4, the commercial agar showed a thermal degradation profile characterized by a sharp peak in the temperature range of 180$290^{\circ} \mathrm{C}$, followed by a broad shoulder and a total mass loss of $67 \%$ at $600^{\circ} \mathrm{C}$. The same degradation peak, although with a wider distribution, was observed for all the agar-based extracts. The maximum of the peak appeared at temperatures of ca. $222^{\circ} \mathrm{C}, 240^{\circ} \mathrm{C}, 234^{\circ} \mathrm{C}$, $250^{\circ} \mathrm{C}$ and $250^{\circ} \mathrm{C}$ for the commercial, HW, HW-US, NaOH+HW and $\mathrm{NaOH}+\mathrm{HW}-\mathrm{US}$ samples, respectively. Although no additional peaks due to the presence of impurities were detected in the HW and HW-US, the onset of the degradation was shifted towards lower temperatures as compared with the commercial and the purified extracts. This suggests that although the presence of impurities reduced slightly the thermal stability of the extracts, since they were bound to a certain fraction of agar, as evidenced by the HPSEC results, their impact was limited. On the other hand, the higher thermal stability of the $\mathrm{NaOH}+\mathrm{HW}$ and $\mathrm{NaOH}+\mathrm{HW}-\mathrm{US}$ extracts as compared to the commercial agar may be related to their greater molecular weight and crystallinity. Additionally, although the molecular weight of the agar fraction was higher for the HW and HW-US extracts, the presence of impurities interacting with the agar impaired its crystalline organization and as a result, reduced slightly the thermal stability of the extracts. The mass loss at $600^{\circ} \mathrm{C}$ was $67 \%$ for the commercial agar, $51 \%$ for $\mathrm{HW}, 45 \%$ for $\mathrm{HW}-\mathrm{US}, 70 \%$ for $\mathrm{NaOH}+\mathrm{HW}$ and $72 \%$ for $\mathrm{NaOH}+\mathrm{HW}-\mathrm{US}$, again proving the presence of inorganic thermally-resistant impurities, such as mineral compounds, in the non-purified extracts. 

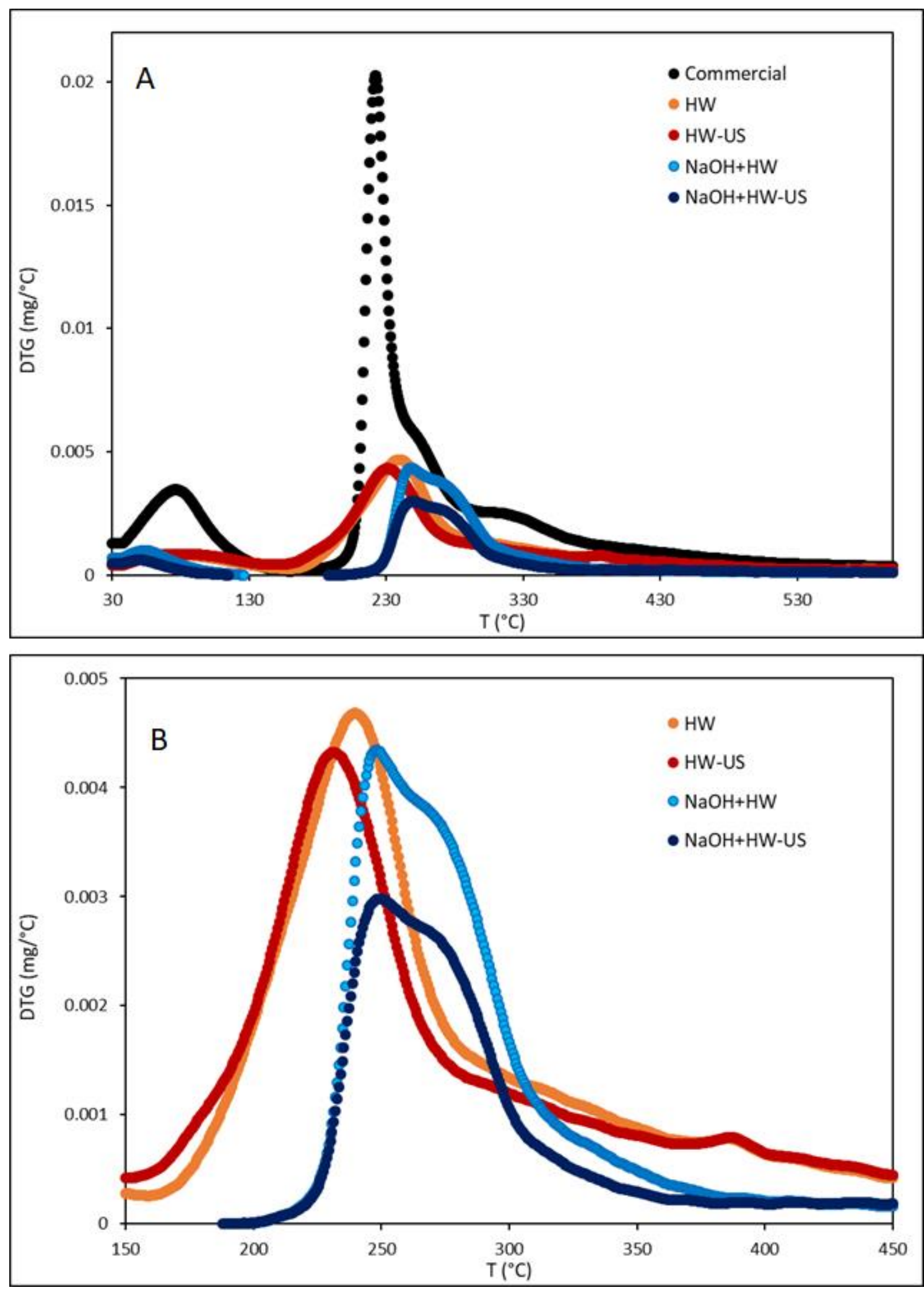

Figure 4. (A) Derivative thermogravimetric (DTG) curves of the agar-based extracts from

Gelidium sesquipedale. (B) Magnification of the temperature range of interest for the produced extracts. 


\subsection{Gels produced from the different agar-based extracts}

The capacity of the produced agar-based extracts to form gels was also evaluated and compared to the commercial agar. All the samples were able to form gels at a concentration of 1.5 wt.- $\%$, which is typically used to produce gels from commercial agars (Kumar \& Fotedar, 2009; Lemus et al., 1991; Yousefi et al., 2013). The visual appearance of the gels is shown in Figure 5A, where it is evidenced that whereas the commercial agar and the purified extracts presented a whitish hue, the non-purified extracts showed a dark brown coloration, most likely due to the presence of polyphenols and proteins. The UV-Vis spectra from solutions of the different extracts (cf. Figure 5B) clearly showed that the non-purified materials presented much higher absorbance values through the whole wavelength range. Several peaks, with maxima located at ca. 290, 330 and $965 \mathrm{~nm}$, were detected in the spectra from all the samples, although they appeared much more defined in the HW and HW-US extracts. These peaks have been previously related to the presence of phenolic compounds (Gallori, Bilia, Bergonzi, Barbosa, \& Vincieri, 2004; Gregoris \& Stevanato, 2010), thus providing further evidence for the higher amount of these compounds in the non-purified extracts. Moreover, although the differences were small, it seems that the extraction of impurities promoted by the sonication process gave rise to slightly greater absorbance values. 

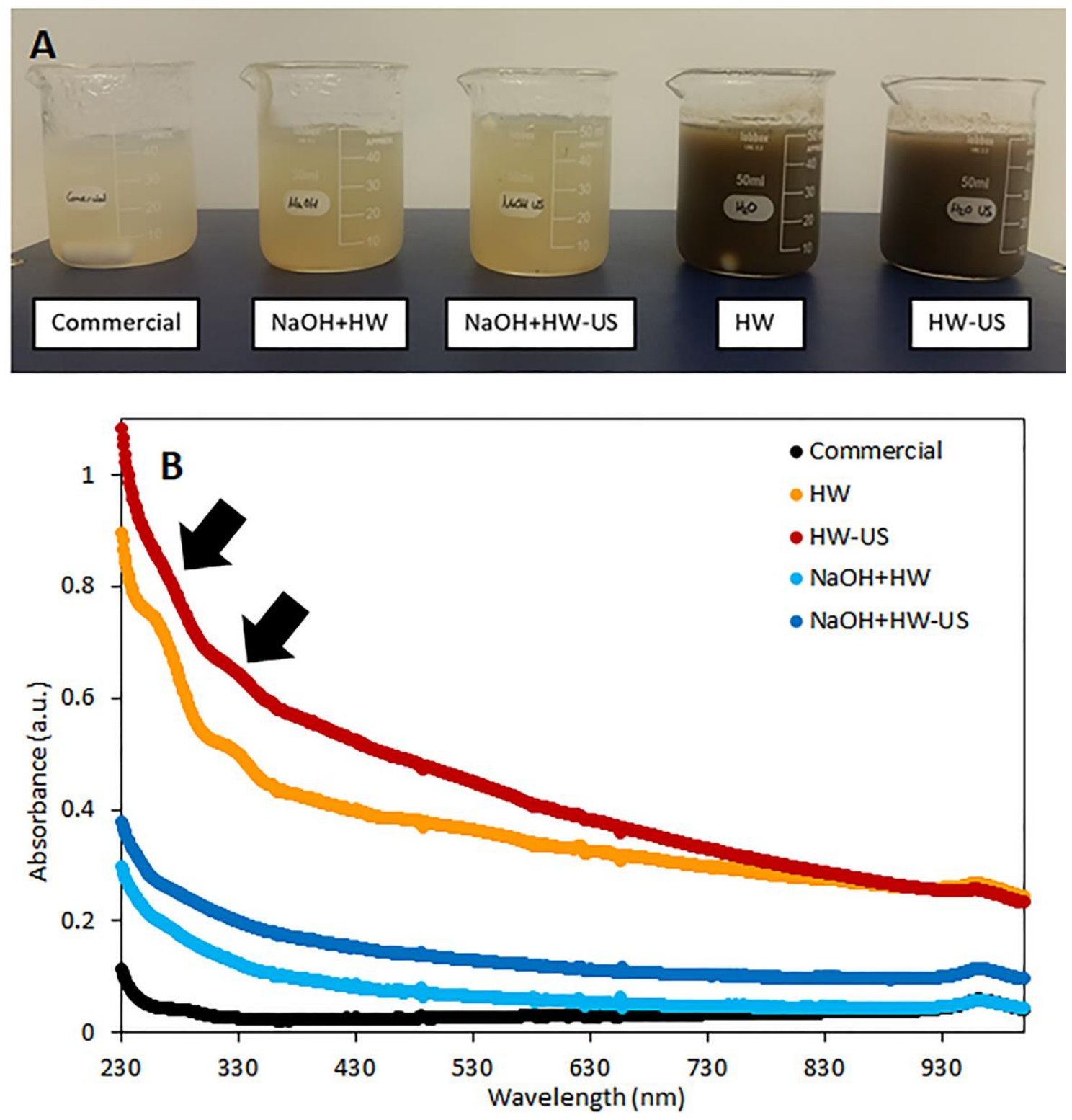

Figure 5. (A) Visual appearance of the gels and (B) UV-Vis spectra from the Gelidium sesquipedale agar-based extracts.

The mechanical properties of the agar-based gels are an important attribute, which will determine their range of applications. Therefore, they were evaluated by means of penetration tests. The calculated gel fracture strength values are gathered in Table 2. It is evident that the gels obtained from the commercial agar and the purified extracts presented significantly greater fracture strength values than the non-purified extracts. This seems to be related to two different factors: (i) the inherent higher agar content in the purified extracts 
and (ii) the higher sulphate content in the non-purified extracts. Similar gel strength values of ca. $1000 \mathrm{~g} / \mathrm{cm}^{2}$ have been reported for agars extracted from Gelidium sesquipedale with sulphate contents ranging from 1.0 to $1.6 \%$ (Mouradi-Givernaud et al., 1999). On the other hand, lower strength values of ca. $138 \mathrm{~g} / \mathrm{cm}^{2}$ and $459 \mathrm{~g} / \mathrm{cm}^{2}$ have been reported for the agars extracted from Gracilaria cliftonii (sulphate content of ca. 8\%) (Kumar \& Fotedar, 2009) and Gracilaria corticata (sulphate content of ca. 2.7\%) (Yousefi et al., 2013), respectively. Additionally, the ability of alkali treatments to remove sulphate groups from the C-6 in the galactose units from agar and the implications of this in increasing the gel strength has already been demonstrated. For instance, the gel strength of the agar extracted from Gelidium rex increased from $590 \mathrm{~g} / \mathrm{cm}^{2}$ to $1272 \mathrm{~g} / \mathrm{cm}^{2}$ when the seaweed was treated with $10 \% \mathrm{NaOH}$ prior to extraction (Matsuhiro \& Urzúa, 1990). The softer gels obtained from the HW and HW-US extracts would be optimum for their application as food ingredients (PereiraPacheco et al., 2007), hence highlighting the potential of the simplified extraction protocols to produce more cost-effective agar-based extracts with interest for the food industry sector.

Table 2. Fracture strength of the gels from the Gelidium sesquipedale agar-based extracts. Data shown as mean $+/-\mathrm{SD}, \mathrm{n}=3$.

\begin{tabular}{|l|c|}
\cline { 2 - 2 } \multicolumn{1}{c|}{} & $\begin{array}{c}\text { Fracture strength } \\
\left(\mathrm{g} / \mathrm{cm}^{2}\right)\end{array}$ \\
\hline Commercial & $1030 \pm 122^{\mathrm{b}}$ \\
\hline $\mathrm{HW}$ & $245 \pm 10^{\mathrm{a}}$ \\
\hline $\mathrm{HW}-\mathrm{NS}$ & $275 \pm 10^{\mathrm{a}}$ \\
\hline $\mathrm{NaOH}+\mathrm{HW}$ & $979 \pm 71^{\mathrm{b}}$ \\
\hline $\mathrm{NaOH}+\mathrm{HW}-\mathrm{US}$ & $1183 \pm 173^{\mathrm{b}}$ \\
\hline
\end{tabular}

Values with different letters are significantly different $(\mathrm{p} \leq 0.05)$. 


\section{Conclusions}

Agar-based extracts from Gelidium sesquipedale have been produced by applying a simple hot-water treatment (HW) and a combined hot water-sonication method (HW-US), with extraction yields of ca. 10-12\%. The combined treatment allowed to reduce the total extraction time by 4-fold, without significantly reducing the extraction yield. In addition, the application of an alkali pre-treatment $(\mathrm{NaOH}+\mathrm{HW}$ and $\mathrm{NaOH}+\mathrm{HW}-\mathrm{US})$ was evaluated for the removal of impurities prior to the extraction, but much lower extraction yields of ca. 2$3 \%$ were attained. While the $\mathrm{NaOH}+\mathrm{HW}$ and $\mathrm{NaOH}+\mathrm{HW}-\mathrm{US}$ extracts were mainly composed of agar, the HW and HW-US contained additional compounds such as proteins, polyphenols and mineral substances, which were responsible for their great antioxidant capacity, higher than that previously reported for the extracts from other Gelidium species. These non-purified HW and HW-US extracts led to the production of brownish gels, with lower gel strength than a commercial agar, being optimum for their application as texture modifiers or thickening agents in food-related applications.

\section{Acknowledgements}

This work was financially supported by the "Agencia Estatal de Investigación" and cofunded by the European Union's Horizon 2020 research and innovation programme (ERANet SUSFOOD2). Marta Martinez-Sanz is recipient of a Juan de la Cierva (IJCI-201523389) contract from the Spanish Ministry of Economy, Industry and Competitiveness. Dr. Patricia López-Sánchez is acknowledged for her valuable help with the characterization of the mechanical properties.

\section{Conflict of interest}

The authors declare that they have no conflict of interest. 


\section{Informed consent, human or animal rights}

No conflicts, informed consent, human or animal rights applicable.

\section{Declaration of authors agreement}

The authors agree to authorship and submission of the manuscript for peer review.

\section{Author contributions}

This research was planned and overseen by M.M.S. and A.L.R. The analysis and interpretation of high performance size exclusion chromatography results was made by L.G.G.M. with the supervision of A.B. The antifungal activity of the agar-based extracts was evaluated by A.R.B. The total carbohydrate and galactose content in the samples were determined by A.M. All authors reviewed the manuscript. M.M.S. takes responsibility for the integrity of the work as a whole.

\section{References}

Adjé, F., Lozano, Y. F., Lozano, P., Adima, A., Chemat, F., \& Gaydou, E. M. (2010). Optimization of anthocyanin, flavonol and phenolic acid extractions from Delonix regia tree flowers using ultrasound-assisted water extraction. Industrial Crops and Products, 32(3), 439-444.

Ahmad, R., Surif, M., Ramli, N., Yahya, N., Nor, A. R. M., \& Bekbayeva, L. (2011). A preliminary study on the agar content and agar gel strength of Gracilaria manilaensis using different agar extraction processes. World Applied Sciences Journal, 15(2), 184-188.

Alehosseini, A., del Pulgar, E.-M. G., Gómez-Mascaraque, L. G., Martínez-Sanz, M., Fabra, M. J., Sanz, Y., . . . Lopez-Rubio, A. (2018). Unpurified Gelidiumextracted carbohydrate-rich fractions improve probiotic protection during storage. LWT - Food Science and Technology, 96, 694-703.

Alvarez-Gómez, F., Korbee, N., \& Figueroa, F. L. (2016). Analysis of antioxidant capacity and bioactive compounds in marine macroalgal and lichenic extracts using different solvents and evaluation methods. Ciencias marinas, 42, 271-288.

Armisen, R. (1995). World-wide use and importance of Gracilaria. Journal of Applied Phycology, 7(3), 231-243. 
Armisen, R., \& Galatas, F. (1987). Production, properties and uses of agar. Production and utilization of products from commercial seaweeds. FAO Fish. Tech. Pap, 288, 1-57.

Arvizu-Higuera, D. L., Rodríguez-Montesinos, Y. E., Murillo-Álvarez, J. I., MuñozOchoa, M., \& Hernández-Carmona, G. (2008). Effect of alkali treatment time and extraction time on agar from Gracilaria vermiculophylla. Journal of Applied Phycology, 20(5), 515-519.

Atef, M., Rezaei, M., \& Behrooz, R. (2014). Preparation and characterization agarbased nanocomposite film reinforced by nanocrystalline cellulose. International Journal of Biological Macromolecules, 70, 537-544.

Aymard, P., Martin, D. R., Plucknett, K., Foster, T. J., Clark, A. H., \& Norton, I. T. (2001). Influence of thermal history on the structural and mechanical properties of agarose gels. Biopolymers, 59(3), 131-144.

Bleakley, S., \& Hayes, M. (2017). Algal proteins: extraction, application, and challenges concerning production. Foods, 6(5), 33.

Carmona, R., Vergara, J. J., Lahaye, M., \& Niell, F. X. (1998). Light quality affects morphology and polysaccharide yield and composition of Gelidium sesquipedale (Rhodophyceae). Journal of Applied Phycology, 10(3), 323.

Chen, H.-M., Zheng, L., \& Yan, X.-J. (2005). The preparation and bioactivity research of agaro-oligosaccharides. Food Technology and Biotechnology, 43(1), 29-36.

Chen, Y. W., Lee, H. V., Juan, J. C., \& Phang, S.-M. (2016). Production of new cellulose nanomaterial from red algae marine biomass Gelidium elegans. Carbohydrate Polymers, 151, 1210-1219.

Dodgson, K., \& Price, R. (1962). A note on the determination of the ester sulphate content of sulphated polysaccharides. Biochemical Journal, 84(1), 106-110.

Faraj, A., Lebbar, T., Debry, G., \& Najim, L. (1987). Protein and amino acids analysis during alimentary-agar extraction from Gelidium sesquipedale. Hydrobiologia, 151(1), 513-522.

Foster, G. G., \& Hodgson, A. N. (1998). Consumption and apparent dry matter digestibility of six intertidal macroalgae by Turbo sarmaticus (Mollusca: Vetigastropoda: Turbinidae). Aquaculture, 167(3), 211-227.

Freile-Pelegrín, Y., Madera-Santana, T., Robledo, D., Veleva, L., Quintana, P., \& Azamar, J. A. (2007). Degradation of agar films in a humid tropical climate: Thermal, mechanical, morphological and structural changes. Polymer Degradation and Stability, 92(2), 244-252.

Freile-Pelegrın, Y., \& Murano, E. (2005). Agars from three species of Gracilaria (Rhodophyta) from Yucatán Peninsula. Bioresource Technology, 96(3), 295302.

Freile-Pelegrín, Y., \& Robledo, D. (1997). Influence of alkali treatment on agar from Gracilaria cornea from Yucatan, Mexico. Journal of Applied Phycology, 9(6), 533-539.

Fu, C., Tian, H., Li, Q., Cai, T., \& Du, W. (2006). Ultrasound-assisted extraction of xyloglucan from apple pomace. Ultrasonics Sonochemistry, 13(6), 511-516.

Fu, Y.-W., Hou, W.-Y., Yeh, S.-T., Li, C.-H., \& Chen, J.-C. (2007). The immunostimulatory effects of hot-water extract of Gelidium amansii via immersion, injection and dietary administrations on white shrimp Litopenaeus vannamei and its resistance against Vibrio alginolyticus. Fish \& Shellfish Immunology, 22(6), 673-685. 
Gallori, S., Bilia, A. R., Bergonzi, M. C., Barbosa, W. L. R., \& Vincieri, F. F. (2004). Polyphenolic constituents of fruit pulp of Euterpe oleracea Mart.(açai palm). Chromatographia, 59(11-12), 739-743.

Gómez-Mascaraque, L. G., Dhital, S., Lopez-Rubio, A., \& Gidley, M. J. (2017). Dietary polyphenols bind to potato cells and cellular components. Journal of Functional Foods, 37, 283-292.

Gómez-Ordóñez, E., \& Rupérez, P. (2011). FTIR-ATR spectroscopy as a tool for polysaccharide identification in edible brown and red seaweeds. Food Hydrocolloids, 25(6), 1514-1520.

Gregoris, E., \& Stevanato, R. (2010). Correlations between polyphenolic composition and antioxidant activity of Venetian propolis. Food and Chemical Toxicology, 48(1), 76-82.

Guerrero, P., Etxabide, A., Leceta, I., Peñalba, M., \& De la Caba, K. (2014). Extraction of agar from Gelidium sesquipedale (Rodhopyta) and surface characterization of agar based films. Carbohydrate Polymers, 99, 491-498.

Hromádková, Z., \& Ebringerová, A. (2003). Ultrasonic extraction of plant materialsinvestigation of hemicellulose release from buckwheat hulls. Ultrasonics Sonochemistry, 10(3), 127-133.

Hromádková, Z., Ebringerová, A., \& Valachovič, P. (2002). Ultrasound-assisted extraction of water-soluble polysaccharides from the roots of valerian (Valeriana officinalis L.). Ultrasonics Sonochemistry, 9(1), 37-44.

Jerman, T., Trebše, P., \& Mozetič Vodopivec, B. (2010). Ultrasound-assisted solid liquid extraction (USLE) of olive fruit (Olea europaea) phenolic compounds. Food Chemistry, 123(1), 175-182.

Jiménez-Escrig, A., Jiménez-Jiménez, I., Pulido, R., \& Saura-Calixto, F. (2001). Antioxidant activity of fresh and processed edible seaweeds. Journal of the Science of Food and Agriculture, 81(5), 530-534.

Kanmani, P., \& Rhim, J.-W. (2014). Antimicrobial and physical-mechanical properties of agar-based films incorporated with grapefruit seed extract. Carbohydrate Polymers, 102, 708-716.

Khan, M. K., Abert-Vian, M., Fabiano-Tixier, A.-S., Dangles, O., \& Chemat, F. (2010). Ultrasound-assisted extraction of polyphenols (flavanone glycosides) from orange (Citrus sinensis L.) peel. Food Chemistry, 119(2), 851-858.

Kumar, V., \& Fotedar, R. (2009). Agar extraction process for Gracilaria cliftonii. Carbohydrate Polymers, 78(4), 813-819.

Lai, M.-F., \& Lii, C.-y. (1997). Rheological and thermal characteristics of gel structures from various agar fractions. International Journal of Biological Macromolecules, 21(1), 123-130.

Lee, J.-H., \& Kim, G.-H. (2015). Evaluation of antioxidant activity of marine algaeextracts from Korea. Journal of Aquatic Food Product Technology, 24(3), 227 240.

Lee, W.-K., Lim, Y.-Y., Leow, A. T.-C., Namasivayam, P., Abdullah, J. O., \& Ho, C.L. (2017). Factors affecting yield and gelling properties of agar. Journal of Applied Phycology, 29(3), 1527-1540.

Lemus, A., Bird, K., Kapraun, D. F., \& Koehn, F. (1991). Agar yield, quality and standing crop biomass of Gelidium serrulatum, Gelidium floridanum and Pterocladia capillacea in Venezuela. Food Hydrocolloids, 5(5), 469-479.

Lignell, Å., \& Pedersén, M. (1989). Agar composition as a function of morphology and growth rate. Studies on some morphological strains of Gracilaria secundata and Gracilaria verrucosa (Rhodophyta). Botanica Marina, 32(3), 219-228. 
Lowry, O. H., Rosebrough, N. J., Farr, A. L., \& Randall, R. J. (1951). Protein measurement with the Folin phenol reagent. Journal of biological chemistry, 193(1), 265-275.

Marinho-Soriano, E., Fonseca, P. C., Carneiro, M. A. A., \& Moreira, W. S. C. (2006). Seasonal variation in the chemical composition of two tropical seaweeds. Bioresource Technology, 97(18), 2402-2406.

Martínez-Abad, A., Giummarella, N., Lawoko, M., \& Vilaplana, F. (2018). Differences in extractability under subcritical water reveal interconnected hemicellulose and lignin recalcitrance in birch hardwoods. Green Chemistry, 20, 2534-2546.

Martínez-Sanz, M., Lopez-Sanchez, P., Gidley, M., \& Gilbert, E. (2015). Evidence for differential interaction mechanism of plant cell wall matrix polysaccharides in hierarchically-structured bacterial cellulose. Cellulose, 22(3),1541-1563.

Matsuhiro, B., \& Urzúa, C. C. (1990). Agars from Gelidium rex (Gelidiales, Rhodophyta). In S. C. Lindstrom \& P. W. Gabrielson (Eds.), Thirteenth International Seaweed Symposium: Proceedings of the Thirteenth International Seaweed Symposium held in Vancouver, Canada, August 13-18, 1989 (pp. 545549). Dordrecht: Springer Netherlands

Mazumder, S., Ghosal, P. K., Pujol, C. A., Carlucci, M. a. J., Damonte, E. B., \& Ray, B. (2002). Isolation, chemical investigation and antiviral activity of polysaccharides from Gracilaria corticata (Gracilariaceae, Rhodophyta). International Journal of Biological Macromolecules, 31(1), 87-95.

Meena, R., Prasad, K., Ganesan, M., \& Siddhanta, A. K. (2007). Superior quality agar from Gracilaria species (Gracilariales, Rhodophyta) collected from the Gulf of Mannar, India. Journal of Applied Phycology, 20(4), 397.

Mohammed, Z. H., Hember, M., Richardson, R., \& Morris, E. (1998). Kinetic and equilibrium processes in the formation and melting of agarose gels. Carbohydrate Polymers, 36(1), 15-26.

Mouradi-Givernaud, A., Hassani, L. A., Givernaud, T., Lemoine, Y., \& Benharbet, O. (1999). Biology and agar composition of Gelidium sesquipedale harvested along the Atlantic coast of Morocco. Hydrobiologia, 398, 391-395.

Murano, E. (1995). Chemical structure and quality of agars from Gracilaria. Journal of Applied Phycology, 7(3), 245-254.

Nakayama, R., Tamura, Y., Kikuzaki, H., \& Nakatani, N. (1999). Antioxidant effect of the constituents of susabinori (Porphyra yezoensis). Journal of the American Oil Chemists' Society, 76(5), 649.

Nishinari, K., \& Watase, M. (1983). Effect of alkali pretreatment on the rheological properties of concentrated agar-agar gels. Carbohydrate Polymers, 3(1), 39-52.

Pereira-Pacheco, F., Robledo, D., Rodríguez-Carvajal, L., \& Freile-Pelegrín, Y. (2007). Optimization of native agar extraction from Hydropuntia cornea from Yucatán, México. Bioresource Technology, 98(6), 1278-1284.

Pereira, L., Sousa, A., Coelho, H., Amado, A. M., \& Ribeiro-Claro, P. J. (2003). Use of FTIR, FT-Raman and 13 C-NMR spectroscopy for identification of some seaweed phycocolloids. Biomolecular Engineering, 20(4), 223-228.

Phan, T. D., Debeaufort, F., Luu, D., \& Voilley, A. (2005). Functional properties of edible agar-based and starch-based films for food quality preservation. Journal of Agricultural and Food Chemistry, 53(4), 973-981.

Prabakaran, P., \& Ravindran, A. D. (2011). A comparative study on effective cell disruption methods for lipid extraction from microalgae. Letters in Applied Microbiology, 53(2), 150-154. 
Prado-Fernández, J., Rodríguez-Vázquez, J. A., Tojo, E., \& Andrade, J. M. (2003). Quantitation of $\kappa-$, $1-$ and $\lambda$-carrageenans by mid-infrared spectroscopy and PLS regression. Analytica Chimica Acta, 480(1), 23-37.

Rath, J., \& Adhikary, S. (2004). Effect of alkali treatment on the yield and quality of agar from red alga Gracilaria verrucosa (Rhodophyta, Gracilariales) occurring at different salinity gradient of Chilika lake. Indian Journal of Geo-Marine Sciences, 33,202-205.

Re, R., Pellegrini, N., Proteggente, A., Pannala, A., Yang, M., \& Rice-Evans, C. (1999). Antioxidant activity applying an improved ABTS radical cation decolorization assay. Free radical biology and medicine, 26(9), 1231-1237.

Rhim, J.-W. (2011). Effect of clay contents on mechanical and water vapor barrier properties of agar-based nanocomposite films. Carbohydrate Polymers, 86(2), 691-699.

Rocha de Souza, M. C., Marques, C. T., Guerra Dore, C. M., Ferreira da Silva, F. R., Oliveira Rocha, H. A., \& Leite, E. L. (2007). Antioxidant activities of sulfated polysaccharides from brown and red seaweeds. Journal of Applied Phycology, 19(2), 153-160.

Singh, S., Gaikwad, K. K., Park, S.-I., \& Lee, Y. S. (2017). Microwave-assisted step reduced extraction of seaweed (Gelidiella acerosa) cellulose nanocrystals. International Journal of Biological Macromolecules, 99, 506-510.

Singleton, V. L., Orthofer, R., \& Lamuela-Raventós, R. M. (1999). [14] Analysis of total phenols and other oxidation substrates and antioxidants by means of folinciocalteu reagent. Methods in enzymology, 299, 152-178.

Sousa-Pinto, I., Murano, E., Coelho, S., Felga, A., \& Pereira, R. (1999). The effect of light on growth and agar content of Gelidium pulchellum (Gelidiaceae, Rhodophyta) in culture. Hydrobiologia, 398(0), 329-338.

Sousa, A. M., Morais, S., Abreu, M. H., Pereira, R., Sousa-Pinto, I., Cabrita, E. J., . . . Gonçalves, M. P. (2012). Structural, physical, and chemical modifications induced by microwave heating on native agar-like galactans. Journal of Agricultural and Food Chemistry, 60(19), 4977-4985.

Sousa, A. M., Souza, H. K., Uknalis, J., Liu, S.-C., Goncalves, M. P., \& Liu, L. (2015). Electrospinning of agar/PVA aqueous solutions and its relation with rheological properties. Carbohydrate Polymers, 115, 348-355.

Souza, B. W. S., Cerqueira, M. A., Bourbon, A. I., Pinheiro, A. C., Martins, J. T., Teixeira, J. A., .. . Vicente, A. A. (2012). Chemical characterization and antioxidant activity of sulfated polysaccharide from the red seaweed Gracilaria birdiae. Food Hydrocolloids, 27(2), 287-292.

Venugopal, V. (2016). Marine polysaccharides: Food applications: CRC Press.

Wang, J., Sun, B., Cao, Y., Tian, Y., \& Li, X. (2008). Optimisation of ultrasoundassisted extraction of phenolic compounds from wheat bran. Food Chemistry, 106(2), 804-810.

Wang, T., Jónsdóttir, R., Kristinsson, H. G., Hreggvidsson, G. O., Jónsson, J. Ó., Thorkelsson, G., \& Ólafsdóttir, G. (2010). Enzyme-enhanced extraction of antioxidant ingredients from red algae Palmaria palmata. LWT - Food Science and Technology, 43(9), 1387-1393.

Watase, M., \& Nishinari, K. (1983). Rheological properties of agarose gels with different molecular weights. Rheologica acta, 22(6), 580-587.

Whitaker, J. R., \& Granum, P. E. (1980). An absolute method for protein determination based on difference in absorbance at 235 and $280 \mathrm{~nm}$. Analytical biochemistry, 109(1), 156-159. 
Wijesekara, I., Pangestuti, R., \& Kim, S.-K. (2011). Biological activities and potential health benefits of sulfated polysaccharides derived from marine algae.

Carbohydrate Polymers, 84(1), 14-21.

Yousefi, M. K., Islami, H. R., \& Filizadeh, Y. (2013). Effect of extraction process on agar properties of Gracilaria corticata (Rhodophyta) collected from the Persian Gulf. Phycologia, 52(6), 481-487.

Yuan, T.-Q., Xu, F., He, J., \& Sun, R.-C. (2010). Structural and physico-chemical characterization of hemicelluloses from ultrasound-assisted extractions of partially delignified fast-growing poplar wood through organic solvent and alkaline solutions. Biotechnology advances, 28(5), 583-593.

Yuan, Y. V., \& Walsh, N. A. (2006). Antioxidant and antiproliferative activities of extracts from a variety of edible seaweeds. Food and Chemical Toxicology, 44(7), 1144-1150.

Yuan, Y. V., Westcott, N. D., Hu, C., \& Kitts, D. D. (2009). Mycosporine-like amino acid composition of the edible red alga, Palmaria palmata (dulse) harvested from the west and east coasts of Grand Manan Island, New Brunswick. Food Chemistry, 112(2), 321-328.

Zhang, M., Zhang, L., Cheung, P. C. K., \& Ooi, V. E. C. (2004). Molecular weight and anti-tumor activity of the water-soluble polysaccharides isolated by hot water and ultrasonic treatment from the sclerotia and mycelia of Pleurotus tuberregium. Carbohydrate Polymers, 56(2), 123-128. 